\title{
Polarization observables in the elastic scattering of protons from ${ }^{4,6,8} \mathrm{He}$
}

\author{
R. Crespo* \\ Departamento de Física, Instituto Superior Técnico, Taguspark, Av. Prof. Cavaco e Silva, Taguspark, PT-2780-990 Porto Salvo, \\ Oeiras, Portugal and Centro de Física Nuclear, Av. Prof. Gama Pinto, 2, 1699, Lisboa, Portugal \\ A. M. Moro \\ Departamento de Física Atómica, Molecular e Nuclear, Universidad de Sevilla Apdo. 1065, E-41080 Sevilla, Spain
}

(Received 15 February 2007; published 21 November 2007)

\begin{abstract}
We have calculated the $p-{ }^{4,6,8} \mathrm{He}$ elastic scattering differential cross section and polarizations at $297 \mathrm{MeV}$ using the Multiple Scattering expansion of the Optical potential (MSO) reaction scattering framework. The role of the core and valence neutrons contribution to the interaction in the description of the elastic scattering observables is analyzed.
\end{abstract}

DOI: 10.1103/PhysRevC.76.054607

PACS number(s): 24.50.+g, 25.40.Cm, 24.70.+s

\section{INTRODUCTION}

The analyzing power of an unstable beam of ${ }^{6} \mathrm{He}$ on a polarized proton target at an energy of $71 \mathrm{MeV} /$ nucleon was measured recently for the first time [1]. It was found that at this energy the polarization changes sign from positive to negative at around $50^{\circ}$ which is in contradiction with some theoretical predictions [2,3]. In [1] a phenomenological optical model analysis of the data was carried out, and it was found that the behavior of the sign change of the polarization could be explained if the radius of the spin orbit component was set to a larger value than the standard value in this mass region. To understand this behavior one should calculate a microscopic optical potential where dynamics and structure are clearly delineated. This can be achieved making use of the Multiple scattering expansion of the Optical potential (MSO) as formulated by Kermann, McManus, and Thaler (KMT) [4] where the single scattering term is given in the impulse approximation by the product of the free nucleon-nucleon amplitude evaluated at the appropriate energy and the target density. This term of the potential does not treat the ${ }^{6} \mathrm{He}$ few-body dynamics. However, one expects that it will provide us with some insight into the behavior of the phenomenological optical potential.

The KMT formalism is valid for proton incident energies in the intermediate energy region $E_{p}=100-500 \mathrm{MeV}$. Differential cross sections, analyzing powers, and spin rotation parameters for elastic scattering of protons on ${ }^{4} \mathrm{He}$ at $297 \mathrm{MeV}$ were measured [5]. Therefore we calculate the elastic scattering of protons from the helium isotopes at this energy.

In Sec. II we will briefly describe the single scattering approximation of the MSO scattering framework. In Sec. III we discuss the structure models used to evaluate the ground state matter density distributions. In Sec. IV we evaluate the elastic scattering differential cross section and polarization observables and conclude in Sec. V.

\footnotetext{
*Raquel.Crespo@tagus.ist.utl.pt
}

\section{MSO}

The proton-nucleus elastic scattering can be described in terms of an optical model (OM) obtained either microscopically, from first principles, or from fitting the data. We will follow in here the multiple scattering expansion of the optical potential in terms of the free nucleon-nucleon transition amplitude $t_{\mathcal{N N}}$ as formulated by KMT [4].

\section{A. Nucleon-nucleon transition amplitude}

The free $N N$ transition amplitude $t_{\mathcal{N N}}\left(\omega, \overrightarrow{\mathcal{K}}^{\prime}, \overrightarrow{\mathcal{K}}\right)$, describing the scattering from two-nucleon states with relative momenta $\overrightarrow{\mathcal{K}}$ and $\overrightarrow{\mathcal{K}}^{\prime}$ for relative energy $\omega$ in their center of mass (c.m.) frame, is related to the anti-symmetrised scattering amplitude matrix elements by

$$
t_{\mathcal{N N}}\left(\omega, \overrightarrow{\mathcal{K}}^{\prime}, \overrightarrow{\mathcal{K}}\right)=-\frac{\hbar^{2}}{4 \pi^{2} \mu_{\mathcal{N N}}} M_{\mathcal{N N}}\left(\omega, \overrightarrow{\mathcal{K}}^{\prime}, \overrightarrow{\mathcal{K}}\right)
$$

where $\mu_{\mathcal{N} \mathcal{N}}$ is the nucleon-nucleon reduced mass.

In the Wolfenstein representation the most general form of the amplitude, consistent with time-reversal, parity, and rotational invariance, is written as

$$
\begin{aligned}
M\left(\omega, \overrightarrow{\mathcal{K}}^{\prime}, \overrightarrow{\mathcal{K}}\right)= & \mathcal{A}+\mathcal{B}\left(\vec{\sigma}_{0} \cdot \hat{n}\right)\left(\vec{\sigma}_{1} \cdot \hat{n}\right)+\mathcal{C}\left(\vec{\sigma}_{0}+\vec{\sigma}_{1}\right) \cdot \hat{n} \\
& +\mathcal{D}\left(\vec{\sigma}_{0} \cdot \hat{m}\right)\left(\vec{\sigma}_{1} \cdot \hat{m}\right)+\mathcal{E}\left(\vec{\sigma}_{0} \cdot \hat{\ell}\right)\left(\vec{\sigma}_{1} \cdot \hat{\ell}\right) \\
& +\mathcal{F}\left[\left(\vec{\sigma}_{0} \cdot \hat{\ell}\right)\left(\vec{\sigma}_{1} \cdot \hat{m}\right)+\left(\vec{\sigma}_{1} \cdot \hat{m}\right)\left(\vec{\sigma}_{0} \cdot \hat{\ell}\right)\right]
\end{aligned}
$$

where the orthogonal set of unit vectors $\left(\hat{n}=(\overrightarrow{\mathcal{K}} \times \overrightarrow{\mathcal{K}})^{\prime}\right) /\left|\overrightarrow{\mathcal{K}} \times \overrightarrow{\mathcal{K}}^{\prime}\right|, \hat{\ell}=\left(\overrightarrow{\mathcal{K}}^{\prime}+\overrightarrow{\mathcal{K}}\right) /\left|\overrightarrow{\mathcal{K}}^{\prime}+\overrightarrow{\mathcal{K}}\right|, \quad$ and $\hat{m}=\hat{\ell} \times \hat{n}$ are defined by the $N N$ scattering plane [6]. The scattering amplitude can also be expressed as a complex function of the energy $\omega$, the momentum transfer $\vec{q}=\left(\overrightarrow{\mathcal{K}}^{\prime}-\overrightarrow{\mathcal{K}}\right)$ and the total momentum $\overrightarrow{\mathcal{Q}}=\left(\overrightarrow{\mathcal{K}}+\overrightarrow{\mathcal{K}}^{\prime}\right) / 2$ of the $N N$ pair in their center of mass frame:

$$
\left\langle\overrightarrow{\mathcal{K}}^{\prime}|M(\omega)| \overrightarrow{\mathcal{K}}\right\rangle=M\left(\omega, \overrightarrow{\mathcal{K}}^{\prime}, \overrightarrow{\mathcal{K}}\right)=M(\omega, \vec{q}, \overrightarrow{\mathcal{Q}}) .
$$

\section{B. Single scattering factorized optical potential}

Let us then consider the scattering of a proton from a nucleus (of mass number $A$ ) assumed to be well described 
by $n$ clusters. Within MSO, as formulated by KMT, the optical potential can be written as an expansion in terms of the free $N N$ transition amplitudes, $t_{p \mathcal{N}}$ (for projectile $p$-struck nucleon $\mathcal{N}$ scattering). In momentum space, the matrix elements of the single scattering term, evaluated in the optimal factorization [7], are given by

$$
\begin{aligned}
\left\langle\vec{k}^{\prime}|U| \vec{k}\right\rangle= & \frac{A-1}{A} \sum_{i=1}^{n}\left[\rho_{p}^{i}(q) \bar{t}_{p p}(\omega, q, Q / 2, \phi)\right. \\
& \left.+\rho_{n}^{i}(q) \bar{t}_{p n}(\omega, q, Q / 2, \phi)\right],
\end{aligned}
$$

where $\rho_{p}^{i}$ and $\rho_{n}^{i}$ are the nuclear matter density distributions for the protons and neutrons respectively for each cluster of the nucleus. Here, $\bar{t}_{p p}\left(\bar{t}_{p n}\right)$ is the spin averaged amplitude for the $p p, p n$ scattering respectively evaluated at the appropriate energy $\omega=\frac{E}{2}$, and $\phi$ is the angle between the vectors $\vec{Q}=\left(\vec{k}^{\prime}+\vec{k}\right) / 2$ and $\vec{q}=\left(\vec{k}^{\prime}-\vec{k}\right)$. In Eq. (4) other spin components of the $N N$ transition amplitudes for the case of proton scattering from nonzero spin clusters are not included.

Higher order effects have been evaluated and shown to reduce the absorption present in the lower partial waves [7].

The optical potential matrix elements can then be written as a sum of a central and spin-orbit contribution

$$
\left\langle\vec{k}^{\prime}|U| \vec{k}\right\rangle=\left\langle\vec{k}^{\prime}\left|U^{c}\right| \vec{k}\right\rangle+i \vec{\sigma} \cdot \vec{n}\left\langle\vec{k}^{\prime}\left|U^{l s}\right| \vec{k}\right\rangle,
$$

with $\sigma$ the spin operator for the projectile, $\vec{n}=\vec{\kappa} \times \vec{\kappa}^{\prime}$ and

$$
\begin{aligned}
\left\langle\vec{k}^{\prime}\left|U^{c}\right| \vec{k}\right\rangle= & \frac{A-1}{A} \frac{\hbar^{2}}{4 \pi^{2} \mu_{\mathcal{N N}}} \sum\left[\rho_{p}^{i}(q) \mathcal{A}_{p p}(\omega, q, Q / 2, \phi)\right. \\
& \left.+\rho_{n}^{i} \mathcal{A}_{p n}(\omega, q, Q / 2, \phi)\right]
\end{aligned}
$$

and the spin-orbit given as

$$
\begin{aligned}
\left\langle\vec{k}^{\prime}\left|U^{l s}\right| \vec{k}\right\rangle= & \frac{A-1}{A} \frac{\hbar^{2}}{4 \pi^{2} \mu_{\mathcal{N N}}} \frac{-i}{\sin \theta_{N A}} \\
& \times \sum\left[\rho_{p}^{i}(q) \mathcal{C}_{p p}(\omega, q, Q / 2, \phi)\right. \\
& \left.+\rho_{n}^{i} \mathcal{C}_{p n}(\omega, q, Q / 2, \phi)\right] .
\end{aligned}
$$

In here, $\theta_{N A}$ is the scattering angle in the nucleon-nucleus center of mass frame. The evaluation of the off-shell central and spin-orbit amplitudes $\mathcal{A}, \mathcal{C} / \sin \phi$, have shown that for $N N$ relative momenta less than $3 \mathrm{fm}^{-1}$ and $50 \mathrm{MeV} \leqslant \omega \leqslant$ $200 \mathrm{MeV}$, they are essentially independent of the variables $\omega$ and $\phi$. It is then a good approximation to take this angle to its on shell value $\phi=\pi / 2$. At this stage the optical potential is still nonlocal.

In the KMT scattering framework, it is necessary to solve the Lippmann-Schwinger equation for the elastic scattering problem, for the potential $U$,

$$
T^{\prime}=U+U G_{0} T^{\prime} .
$$

The transition amplitude for elastic scattering, $T$, is related to the transition amplitude associated with the potential $U$, through the relation $T=\frac{A}{A-1} T^{\prime}$, with $T^{\prime}=T^{\prime}(U)$ where $T^{\prime}(U)$ is defined in Eq. (8).

\section{STRUCTURE}

\section{A. ${ }^{4} \mathrm{He}$}

To describe the ${ }^{4} \mathrm{He}$ ground state we take a single particle harmonic oscillator model (HO), with parameter $b_{4}=\sqrt{2 / 3}\left\langle r^{2}\right\rangle_{4}^{1 / 2}$. The matter density normalized to the number of nucleons is given has

$$
\rho^{4}(q)=\rho_{n}^{4}(q)+\rho_{p}^{4}(q) .
$$

We assume the same matter density distribution for protons and neutrons,

$$
\rho_{n, p}^{4}(q)=2 \exp \left(-b_{4}^{2} q^{2} / 4\right), \quad b_{4}=1.396 \mathrm{fm},
$$

where we have taken $\left\langle r^{2}\right\rangle_{4}^{1 / 2}=1.71 \mathrm{fm}$.

\section{B. ${ }^{6} \mathrm{He}$}

To describe the ${ }^{6} \mathrm{He}$ structure we consider two models: a few-body model and a harmonic oscillator model. In the former, the ${ }^{6} \mathrm{He}$ is described as a three-body system $n+n+{ }^{4} \mathrm{He}$. The bound wave function is obtained by solving the Schrödinger equation in hyperspherical coordinates with an effective three-body (3B) potential, which is introduced to overcome the underbinding caused by the other closed channels, most important of which the $t+t$ breakup. The $n-{ }^{4} \mathrm{He}$ potential is taken from Refs. [8,9], and use the GPT $N N$ potential [10] with spin-orbit and tensor components. In the model (R5) we consider here the 3B effective potential is described in [11]. The model predicts, with an $\alpha$ particle rms matter radius of $1.49 \mathrm{fm}$, an ${ }^{6} \mathrm{He}$ rms matter radius of $2.50 \mathrm{fm}$. The total wave function is a sum of the three components, $\Psi=\Psi_{12}+\Psi_{c 1}+\Psi_{c 2}$, where 1 and 2 represent the halo neutrons and $\mathrm{c}$ the core. Neutron antisymmetrization implies that $\Psi_{c 2}$ and $\Psi_{c 1}$ are related by permutation of labels, and

$$
\Psi=\bar{\Psi}_{12}\left(\vec{r}_{12}, \vec{r}_{(12) c}\right)+(1+P) \bar{\Psi}_{c 1}\left(\vec{r}_{c 1}, \vec{r}_{(c 1) 2}\right) .
$$

The total wave function $\Psi$ can be transformed into either set of coordinates, so that

$$
\Psi=\bar{\Psi}_{12}\left(\vec{r}_{12}, \vec{r}_{(12) c}\right)=\bar{\Psi}_{c 1}\left(\vec{r}_{c 1}, \vec{r}_{(c 1) 2}\right) .
$$

The one particle density can be written

$$
\rho_{F B}^{6}(r)=\hat{\rho}^{c}(r)+\rho_{n}^{v}(r),
$$

where $\hat{\rho}^{c}(r)$ and $\rho_{n}^{v}(r)$ are the contributions from the core and valence neutrons in the center of mass of the whole nucleus. It follows that the valence neutron density is

$$
\rho_{n}^{v}(r)=2\left(\frac{A}{A-1}\right)^{3} \int d \vec{r}_{c 1}\left|\bar{\Psi}_{c 1}\left(\vec{r}_{c 1}, \frac{A}{A-1} \vec{r}\right)\right|^{2}
$$

and, assuming that the core internal density is $\rho^{c}(r)$, then $\hat{\rho}^{c}(r)$ is obtained by folding with $\rho^{\text {c.m. }}(r)$, the density distribution for the motion of the core center of mass, i.e.,

$$
\hat{\rho}^{c}(r)=\int d \vec{r}_{c} \rho^{c}\left(\vec{r}-\vec{r}_{c}\right) \rho^{\mathrm{c} . \mathrm{m} .}\left(\vec{r}_{c}\right)
$$


where

$$
\rho^{\text {c.m. }}\left(\vec{r}_{c}\right)=\left(\frac{A}{2}\right)^{3} \int d \vec{r}_{12}\left|\bar{\Psi}_{12}\left(\vec{r}_{12}, \frac{A}{2} \vec{r}_{c}\right)\right|^{2} .
$$

In momentum space,

$$
\rho_{F B}^{6}(q)=\hat{\rho}^{c}(q)+\rho_{n}^{v}(q),
$$

where

$$
\hat{\rho}^{c}(q)=\rho^{c}(q) \times \rho^{\mathrm{c} . \mathrm{m} \cdot}(q) .
$$

Here, the core density is

$$
\rho^{c}(q)=\rho_{n}^{c}(q)+\rho_{p}^{c}(q),
$$

with

$$
\rho_{n, p}^{c}(q)=2 \exp \left(-\bar{b}_{4}^{2} q^{2} / 4\right), \quad \bar{b}_{4}=1.216 \mathrm{fm},
$$

where we have taken a point density distribution of rms matter radius of $1.49 \mathrm{fm}$.

We also consider the case where the valence nucleons are described within the harmonic oscillator single particle model. In momentum space these densities are

$$
\rho_{\mathrm{HO}}^{6}(q)=\breve{\rho}^{c}(q)+\breve{\rho}_{n}^{v}(q),
$$

with

$$
\begin{gathered}
\breve{\rho}^{c}(q)=\breve{\rho}_{n}^{c}(q)+\breve{\rho}_{p}^{c}(q) \\
\breve{\rho}_{n, p}^{c}(q)=2 \exp \left(-\breve{b}_{4}^{2} q^{2} / 4\right),
\end{gathered}
$$

and

$$
\breve{\rho}_{n}^{v}(q)=2\left(1-\breve{b}_{v}^{2} q^{2} / 6\right) \exp \left(-\breve{b}_{v}^{2} q^{2} / 4\right) .
$$

The range parameters are chosen to reproduce the rms radius of ${ }^{6} \mathrm{He}$, i.e.,

$$
\left\langle r^{2}\right\rangle_{6}=\breve{b}_{4}^{2}+\frac{5}{6} \breve{b}_{v}^{2} .
$$

We assume $\left\langle r^{2}\right\rangle_{6}^{1 / 2}=2.5 \mathrm{fm}$ and take two sets of parameters: $\breve{b}_{4}=1.5 \mathrm{fm}$ and $\breve{b}_{v}=2.1 \mathrm{fm}$ (HO1) and $\breve{b}_{4}=1.396 \mathrm{fm}$ and $\breve{b}_{v}=2.27 \mathrm{fm}(\mathrm{HO} 2)$. In the second set (HO2) the parameters for the $\alpha$ core are the same than those for the ${ }^{4} \mathrm{He}$ particle.

In Fig. 1 the contributions to the matter density distributions of ${ }^{6} \mathrm{He}$ are compared with that of the neutron/proton ${ }^{4} \mathrm{He}$ matter distribution (thin dark solid line). The solid light (dashed light) curve represents the neutron valence density distribution within the few body (HO1) model. The thick solid dark (dashed dark) curve represents the core density distribution within the few body (HO1) model. The valence neutrons density distribution is shorter ranged in momentum space configuration. Due to the density distribution of the c.m. motion the core contribution is shorter ranged when compared with the ${ }^{4} \mathrm{He}$ matter density distribution.

\section{C. ${ }^{8} \mathrm{He}$}

To describe the ${ }^{8} \mathrm{He}$ ground state we take the cluster orbital shell model approximation (COSMA) wave function proposed by Zhukov et al. [12]. In this work a simple parametrized Gaussian form for the core and valence nucleon densities

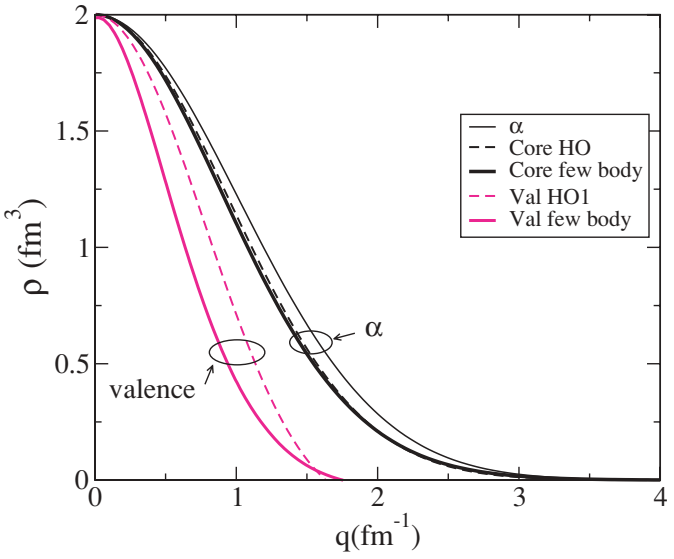

FIG. 1. (Color online) Calculated neutron/proton matter density distributions for ${ }^{4,6} \mathrm{He}$. The thin dark solid line represents the neutron/proton matter distribution of ${ }^{4} \mathrm{He}$. The solid light (dashed light) curve corresponds to the neutron valence density distribution within the few body (HO1) model. The thick solid dark (dashed dark) curve represents the core density distribution within the Few body (HO1) model.

is also presented. When compared with the full COSMA model approximation this density produces the same rms matter radius and accurate values for $r_{v}=3.14 \mathrm{fm}$ and $r_{p}=$ $1.69 \mathrm{fm}$, the mean distances of the valence neutrons, and of a point proton from the ${ }^{8} \mathrm{He}$ c.m., respectively. The Fourier transforms of these densities are

$$
\check{\rho}_{n, p}^{c}(q)=2 \exp \left(-\breve{b}_{4}^{2} q^{2} / 4\right), \quad \check{b}_{4}=1.38 \mathrm{fm}
$$

for the $\alpha$ core cluster, and

$$
\begin{aligned}
\check{\rho}_{n}^{v}(q) & =4\left(1-\check{b}_{v}^{2} q^{2} / 6\right) \exp \left(-\check{b}_{v}^{2} q^{2} / 4\right), \\
\check{b}_{v} & =1.99 \mathrm{fm}
\end{aligned}
$$

for the valence neutrons cluster.

\section{RESULTS}

In this section we evaluate the elastic scattering differential cross section and polarization for the scattering of protons on ${ }^{4,6,8} \mathrm{He}$ at $E_{\text {lab }}=297 \mathrm{MeV}$ using the multiple scattering expansion of the optical potential MSO. In the impulse optimal factorization of the single scattering approximation the optical potential can be written as a product of target densities and off-shell free $N N$ transition amplitudes evaluated at the appropriate energy. These amplitudes were obtained from a realistic $N N$ Paris interaction, as in [7]. The Coulomb interaction was included in an approximate way using the subtracted transition amplitude method $[7,13]$ with an uniform charge sphere.

In Fig. 2 we show the differential cross section (upper figure) and analyzing power (lower figure) for protons on ${ }^{4} \mathrm{He}$ at $297 \mathrm{MeV}$. The solid line represents the calculated cross section for protons on ${ }^{4} \mathrm{He}$ as described in the text. When compared with the data taken from [5], one sees that the calculated observable reproduces reasonably well the small angular region but decays faster than the data. This is probably 

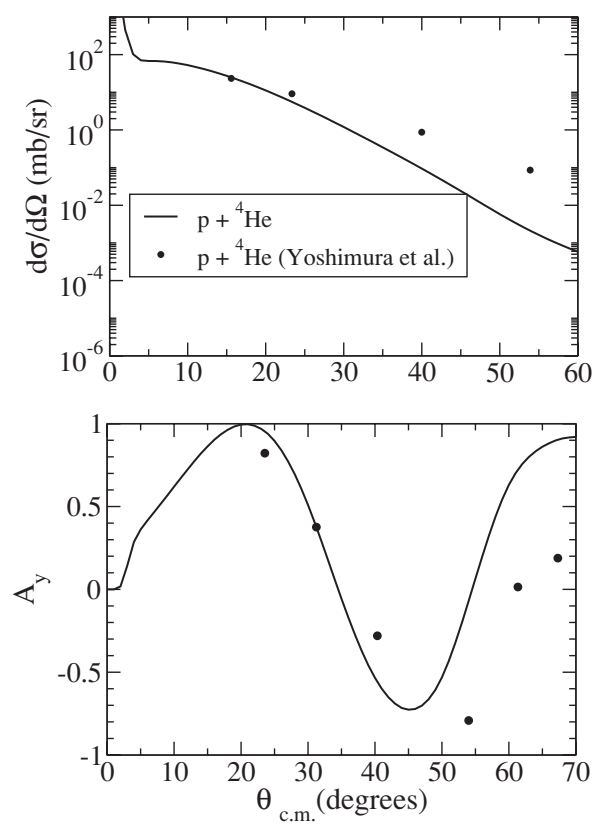

FIG. 2. Calculated elastic differential cross section for $p-{ }^{4} \mathrm{He}$ at $297 \mathrm{MeV}$. The data are for $p-{ }^{4} \mathrm{He}$ [5].

due to the breakdown at large angles of the KMT mean field theory for such a light nucleus like ${ }^{4} \mathrm{He}$.

In the upper part of Fig. 3 we compare the differential cross section for protons on ${ }^{4,6} \mathrm{He}$ isotopes at $297 \mathrm{MeV}$. The calculated differential cross section for proton on ${ }^{6} \mathrm{He}$ using the few-body (thick solid line) and harmonic oscillator HO1 (open circles) densities are bigger than that of $p-{ }^{4} \mathrm{He}$ at small
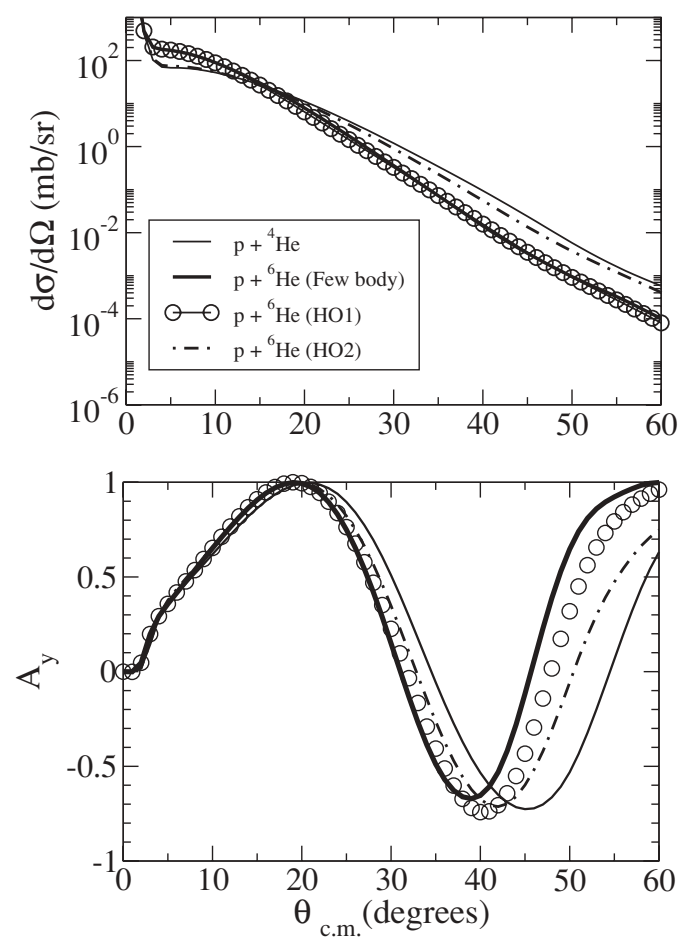

FIG. 3. Calculated elastic differential cross section for $p-{ }^{4,6} \mathrm{He}$ at $297 \mathrm{MeV}$ using different structure models for ${ }^{6} \mathrm{He}$. angles where the valence neutron contribution is crucial and are identical to each other specially at large angles. This is due to the fact that in this angular region the differential cross section essentially probes the core contribution to the optical potential, and this is essentially the same in both models. In addition, the differential cross sections calculated with both the $\mathrm{FB}$ and $\mathrm{HO} 1$ structure models decay faster than that of proton scattering from ${ }^{4} \mathrm{He}$. This follows from the fact that in both models the matter density distribution of the core in momentum space is shorter ranged than that of ${ }^{4} \mathrm{He}$. In fact, the $\mathrm{HO} 2$ model has a similar core contribution than that of the ${ }^{4} \mathrm{He}$ and therefore the calculated differential cross section, represented by the dashed-dotted line, decays more slowly. Therefore at large angles the differential cross section essentially probe that structure part of information contained in the core contribution to the optical model.

In the lower part of Fig. 3 we show the calculated analyzing power for $p^{4,6} \mathrm{He}$. In both cases the observable changes from positive to negative sign at this energy as for the case at lower energy [5]. At small angles the calculated observable for proton on ${ }^{6} \mathrm{He}$ using the few body and the HO1 model are the same despite the valence matter density distributions being different. This is due to the fact that the spin-orbit contribution from the halo valence neutrons is very small. In fact, the short range character of the valence halo density distribution when folded with the spin-orbit component of the $N N$ scattering amplitude, which approaches zero at small momentum transfer, gives a negligible contribution to the overall spin-orbit force. The $p-{ }^{6} \mathrm{He}$ polarization is slightly shifted inwards when compared to the $p-{ }^{4} \mathrm{He}$ polarization.

In the upper (lower) part of Fig. 4 the dashed line represents the elastic scattering differential cross section (polarization) for $p-{ }^{8} \mathrm{He}$. At very small angles, where the valence neutrons contribution is important, the differential cross section is larger than that of $p{ }^{4,6} \mathrm{He}$. At larger angles it decreases slowly than
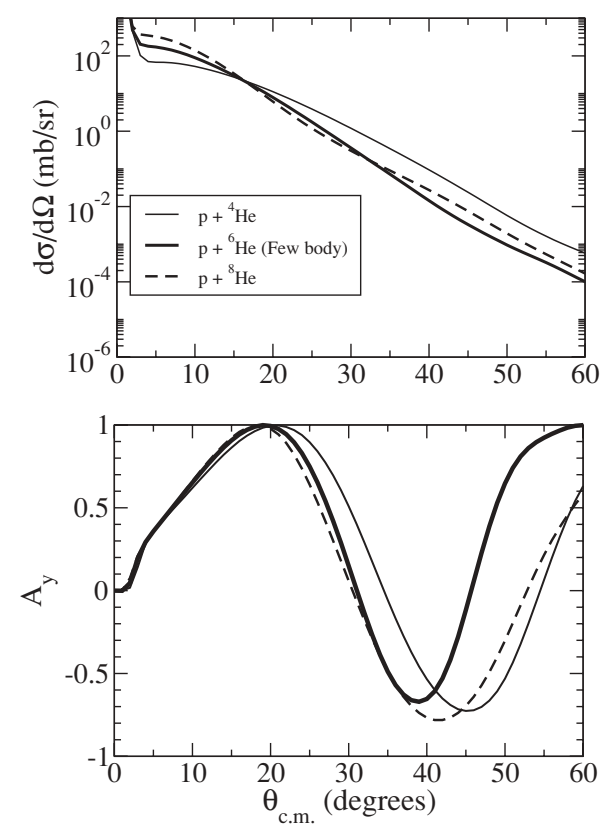

FIG. 4. Elastic differential cross section for $p{ }^{4,6,8} \mathrm{He}$ at $297 \mathrm{MeV}$. 
that of the $p-{ }^{6} \mathrm{He}$ because the core contribution to the optical potential is longer ranged in momentum space. Although the core contribution to the optical potential is identical to that of the ${ }^{4} \mathrm{He}$ the skin of the valence neutrons have here a mass effect in shifting the analyzing power slightly inwards. Nevertheless one can say that the analyzing powers for $p^{-4,6,8} \mathrm{He}$ are very similar.

\section{CONCLUSIONS}

We have studied the elastic scattering of protons from ${ }^{4,6,8} \mathrm{He}$ and calculated the differential cross section and analyzing power elastic observables. These calculations were performed making use of the impulse approximation to the single scattering term of the multiple scattering expansion of the optical potential. In this approach dynamics and structure are clearly delineated. We have evaluated the differential cross section and analyzing power observables. We have shown that the differential cross section probe that structure part of information contained in the core contribution to the optical model. We have also shown that the the spin-orbit contribution from the halo valence neutrons is very small and does not contribute to the analyzing power. Therefore, the long-range halo contribution cannot justify a significant modification of the spin-orbit potential (in particular, the increase of the potential radius), as suggested in the phenomenological analysis done in Ref. [1].

In addition, we have found that the polarization observable for proton $+{ }^{6} \mathrm{He}$ changes sign from positive to negative at around $30^{\circ}$, and that the analyzing power for $p^{-4,6,8} \mathrm{He}$ are very similar.

Experimental data for these reactions would be very useful to assess the validity of the conclusions outlined in this work. Preliminary results have been already obtained at RIKEN for proton $-{ }^{8} \mathrm{He}[14]$.

\section{ACKNOWLEDGMENTS}

The financial support of Fundação para a Ciência e a Tecnologia from grant Nos. POCTI/FNU/43421/2001 is acknowledged. A.M.M. acknowledges the financial support of Junta de Andalucía.
[1] M. Hatano et al., Eur. Phys. J. A 25, 255 (2005).

[2] S. P. Weppner, O. Garcia, and Ch. Elster, Phys. Rev. C 61, 044601 (2000).

[3] D. Gupta, C. Samanta, and R. Kanungo, Nucl. Phys. A674, 77 (2000).

[4] A. K. Kerman, H. McManus, and R. M. Thaler, Ann. Phys. (NY) 8, 551 (1959).

[5] M. Yoshimura et al., Phys. Rev. C 63, 034618 (2001).

[6] L. Wolfenstein and J. Askin, Phys. Rev. 85, 947 (1952).

[7] R. Crespo, R. C. Johnson, and J. A. Tostevin, Phys. Rev. C 44, R1735 (1991); 46, 279 (1992).

[8] J. Bang and C. Gignoux, Nucl. Phys. A313, 119 (1979).
[9] I. J. Thompson, B. V. Danilin, V. D. Efros, J. S. Vaagen, J. M. Bang, and M. V. Zhukov, Phys. Rev. C 61, 024318 (2000).

[10] P. Pires, D. Gogny, and R. de Tourreil, Phys. Lett. B32, 591 (1970).

[11] B. V. Danilin, I. J. Thompson, M. V. Zhukov, and J. S. Vaagen, Nucl. Phys. A632, 383 (1998).

[12] M. V. Zhukov, A. A. Korsheninnikov, and M. H. Smedberg, Phys. Rev. C 50, R1 (1994).

[13] R. Crespo and J. A. Tostevin, Phys. Rev. C 41, 2615 (1990).

[14] S. Sakaguchi, Contribution to The International Symposium on Physics of Unstable Nuclei, ISPUN07, Vietnam, 2007. 